\title{
Job Scheduling in Grid Computing with Fast Artificial Fish Swarm Algorithm
}

\author{
M. A. Awad El- \\ bayoumy \\ Faculty of Computer \\ \& Information \\ Mansoura University, \\ Egypt
}

\author{
M. Z. Rashad \\ Faculty of Computer \\ \& Information \\ Mansoura University, \\ Egypt
}

\author{
M. A. Elsoud \\ Faculty of Computer \\ \& Information \\ Mansoura University, \\ Egypt
}

\author{
M. A. El-dosuky \\ Faculty of Computer \\ \& Information \\ Mansoura University, \\ Egypt
}

\begin{abstract}
One of the problems in grid computing is job scheduling. It is known that the job scheduling is NP-complete, and thus the use of heuristics is the de facto approach to deal with this practice in its difficulty. The proposed is an apply FAFSA in job scheduling and comparison between FASFA and normal AFSA
\end{abstract}

\section{Keyword}

AFSA, Levy, Fast Artificial Fish Swarm Algorithm, FAFSA

\section{INTRODUCTION}

Grid computing[1][2] is a type of distributed computing that work to divide computing, applications, and data storage, or network resources between organizations and geographical spread, And that its road to change the method of dealing with complex computational problems. In a large scale computing systems such as grid computing systems, there are often large amounts of resources available to be used for computing jobs. Since these resources can cost many millions of dollars to achieve the maximum made? use of an important problem. Scheduling in a grid computing [3] [4] system is not as simple as scheduling on a multiprocessor system because of several factors. These factors include the fact that grid resources are sometimes used by paying customers who have interest in how their jobs are being scheduled. Also, grid computing systems usually operate in very remote locations Task Scheduler [5] for groups can occur across a network. Because of these reasons considering scheduling in grid computing is interesting and important problem to examine.

\section{PREVIOUS WORK}

Genetic Algorithm (GA)[6] is a search heuristic that mimics the process of natural selection. This heuristic is routinely used to generate useful solutions to optimization and search problems. Advantage it solves problems with multiple solutions, Genetic algorithm is a method which is very easy to understand and it practically does not demand the knowledge of mathematics. Disadvantage There is no absolute assurance that a genetic algorithm will find a global optimum. It happens very often when the populations have a lot of subjects.

Ant Colony Optimization algorithm (ACO) [7]: is a probabilistic technique for solving computational problems which can be reduced to finding good paths through graphs.

Advantage Inherent parallelism, Efficient for Traveling Salesman Problem and similar problems, Can be used in dynamic applications
Disadvantage Theoretical analysis is difficult, Sequences of random decisions (not independent), Probability distribution changes by iteration

\section{ARTIFICIAL FISH SWARM ALGORITHM}

The basic idea of AFSA [11] [12] is simulated fish behaviors such as swarming, preying, following with local search of fish individual for reaching the global optimum; it is random and parallel search algorithm.

\subsection{Some definitions and concept}

Mathematical models imitate the fish swarm series of behavior in nature which can be defined as [13]:
1. Random behavior
2. Searching behavior
3. Swarming behavior
4. Chasing behavior
5. Leaping behavior

The next behavior of artificial fish depends on its current state and environmental state. Random behavior can be presented as the initialization phase of the algorithm.[14]

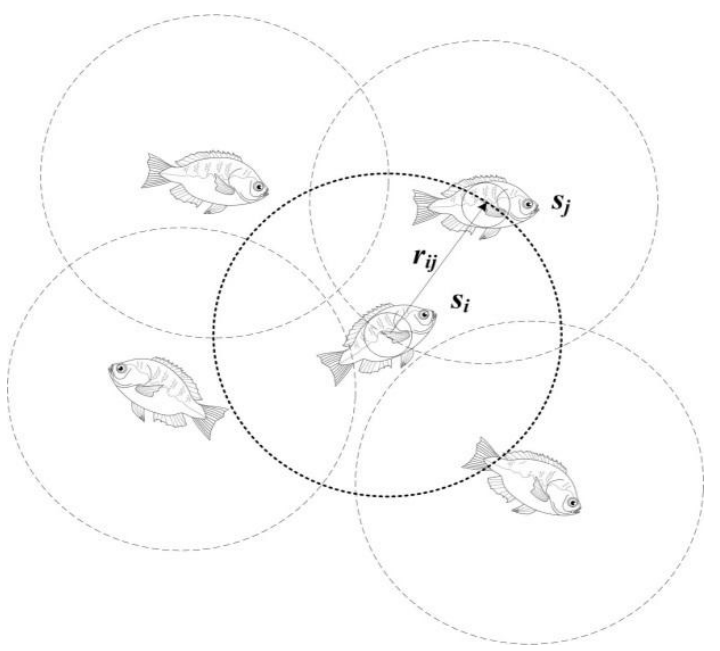

Figure 1 Vision concepts of Artificial Fish

The crucial step in the AFS algorithms is a "visual scope". A basic biological behavior of any animal is to discover a region with more food, by vision or sense. Depending on the current position of the individual in the population, marked as $x^{i} \in$ $\mathrm{Rn}$, three possible situations may occur [16]: 
1. When the "visual scope" is empty, and there are no other individuals in its neighborhood to follow, $\mathrm{x}^{\mathrm{i}}$ individual moves randomly searching for a better region

2. When the "visual scope" is crowded, the $x^{i}$ individual has difficulty to follow any particular individual, and searches for a better region choosing randomly another location from the "visual scope".

3. When the "visual scope" is not crowded, the $x^{\mathrm{i}}$ individual can choose between two options: to swarm moving towards the central or to chase moving towards the best location. The condition that determines the crowd issue of $x^{i}$ individual in the 'visual scope' is given in

$$
\frac{n p^{i}}{m} \leq \theta
$$

where $\theta \in(0,1]$ is the crowd parameter, $m$ is the number of individuals in the population and $n p^{i}$ is the number of individuals in the "visual scope". In the searching behavior phase, an individual is randomly chosen in the "visual scope" of $\mathrm{x}^{\mathrm{i}}$ and a movement towards it is carried out if it improves current $x^{i}$ location. Otherwise, the $x i$ individual moves randomly. The swarming behavior is characterized by a movement towards the central point in the "visual scope" of $x^{i}$. The swarming behavior is progressive stage that is activated only if the central point has a better function value than the current $x^{i}$. Otherwise, the point $x^{i}$ follows the searching behavior. The chasing behavior presents a movement towards the point that has the last function value, xmin. The swarm and chase behavior can be considered as local search. Leaping behavior solves the problem when the best objective function value in the population does not change for a certain number of iterations. In this case the algorithm selects random individual from the population. This process empowers algorithm for obtaining better results in solving numerous problems.

\subsection{Artificial fish-swarm algorithm}

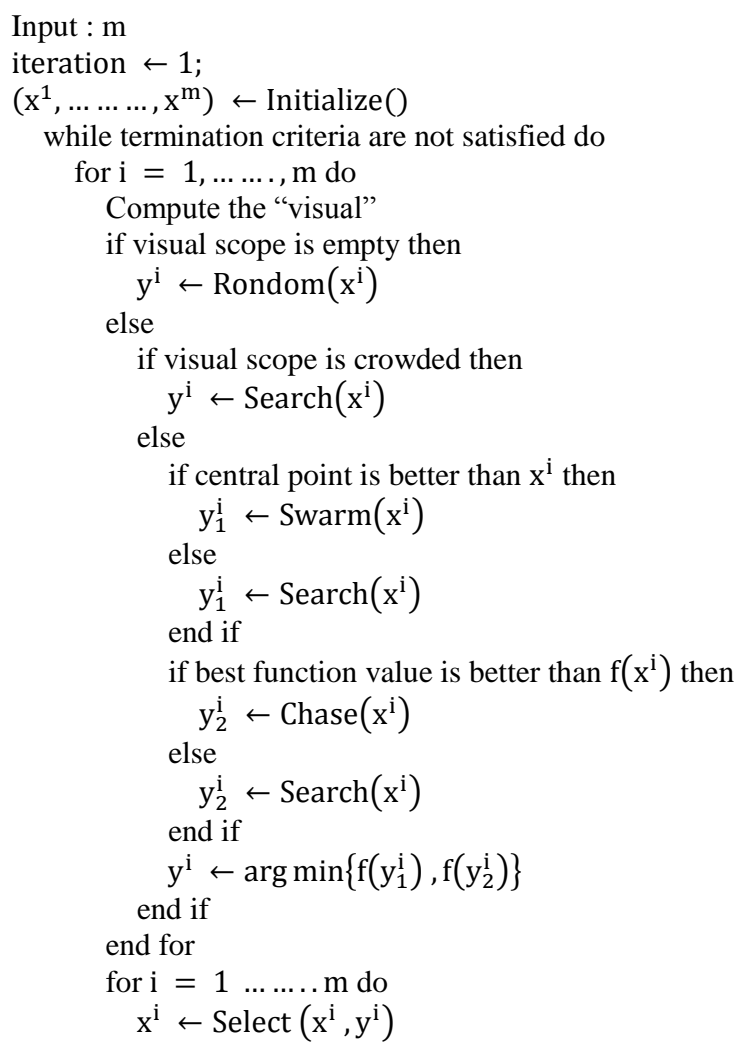

end for

if iteration $>m$ then

if "stagnation" occurs then

Randomly Choose a point $\mathrm{x}^{\mathrm{i}}$

$\mathrm{y}^{\mathrm{i}} \leftarrow$ Leap $\left(\mathrm{x}^{\mathrm{i}}\right)$

end if

end if

end while

iteration $\leftarrow$ iteration +1

\section{THE FAST ARTIFICIAL FISH-} SWARM ALGORITHM (FAFSA)

In the AFSA, there are many parameters that have impacts on the final optimization result, in this paper; we only consider the parameter Rand $O$ we will use Brownian motion and Levy flight Algorithms

\subsection{Definition of Levy Flight}

A Levy flight [18] is the random walk [19] a move lengths probability distribution that heavy tail. When know as walk away in an area larger than the distance of one, the steps that are in random directions isotropic

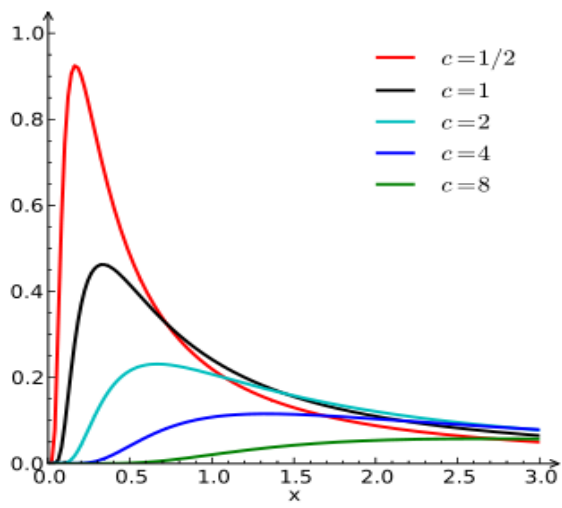

Figure 2: Levy Flight

Definition:

The probability density function of the Levy distribution over the domain $X \geq \mu$ is

$$
f(x ; \mu, c)=\sqrt{\frac{c}{2 \pi}} \frac{e^{-\frac{c}{2(x-\mu)}}}{(x-\mu)^{3 / 2}}
$$

Where $\mu$ is the location parameter and $\mathrm{c}$ is the scale parameter. The cumulative distribution function is

$$
\mathrm{F}(\mathrm{x} ; \mu, \mathrm{c})=\operatorname{erfc}\left(\sqrt{\frac{\mathrm{c}}{2(\mathrm{x}-\mu)}}\right)
$$

Where $\operatorname{erfc}(\mathrm{z})$ is the complementary error function? The shift parameter $\mu$ has the effect of shifting the curve to the right by an amount $\mu$, and changing the support to the interval $[\mu, \infty)$. Like all stable distributions, the Levy distribution has a standard form $\mathrm{f}(\mathrm{x} ; 0,1)$ which has the following property:

$$
f(x ; \mu, c) d x=f(y ; 0,1) d y
$$

Where $y$ is defined as

$$
y=\frac{x-\mu}{c}
$$


In original AFSA, there are many parameters that have impacts on the final optimization result. In order to elevate the global convergence of AFSA, one of this parameter is random behavior. The major contribution in the work is to merge the Levy flight algorithm for randomization in the original AFSA

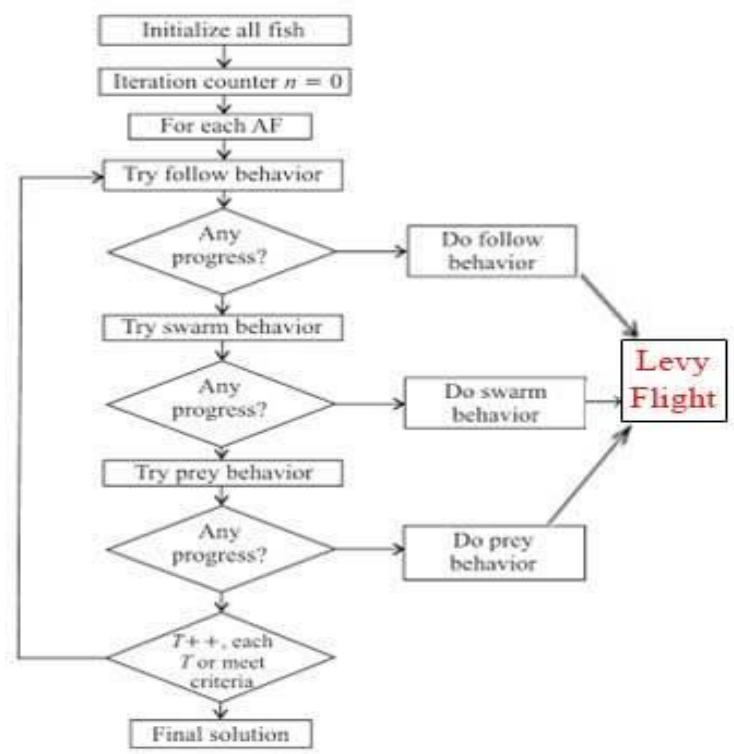

\section{CONCLUSION}

In this paper, we develop a novel method by introducing the modified AFSA for job scheduling in gird computing. AFSA is novel method to search global optimal value by AF`s prey behavior, swarming behavior and following behavior. Therefore, we modified the AFSA with adding levy algorithm to adaptive random behavior. Then we have used FAFSA to introduce a novel method for job scheduling. We evaluate the performance of our method and compared it with normal FS algorithms, under the same condition. From the simulated experiment the result of FAFSA is better than others. First we tested a small scale job scheduling problem involving 2 resources and 10 jobs represented as $(2,10)$. The resource speeds of the 2 resources are 3,2 CPU TIME, and the job length of 10 jobs are $8,12,16,20,24,28,30,36,40$ cycles, respectively.

Table.1 shows the results (makespan) for 5 AFSA runs, FASFA runs. The optimal result is supposed to be 43 . While AFSA provided the best results once, and

FASFA provided the best result three times.

We tested the AFSA and FASFA for large scale job scheduling. Table. 2 shows the results. We can see that the performance of FASFA is much better than normal AFSA under the same condition.

Figure 3 FASFA

Table 1: The results for 5 AFSA runs, and 5 FASFA runs

\begin{tabular}{|c|c|c|c|c|c|c|c|}
\hline Method & 1 & 2 & 3 & 4 & 5 & Average makespan & Best makespan \\
\hline AFSA & 43.6 & 43 & 43.33 & 44 & 43.72 & 43.53 & 43 \\
\hline FASFA & 43 & 43.1 & 43 & 43.3 & 43 & 43.26 & 43 \\
\hline
\end{tabular}

Table 2: Run time and performance comparison

\begin{tabular}{|c|c|c|c|c|c|c|c|}
\hline \multicolumn{8}{|c|}{ (Job, Resource) } \\
\hline $\begin{array}{l}\text { Metho } \\
\text { d }\end{array}$ & $(100,10)$ & $(200,15)$ & $(200,30)$ & $(300,15)$ & $(300,30)$ & $(500,30)$ & $(500,50)$ \\
\hline FSA & $\begin{array}{l}\text { Makespan:120 } \\
.3 \\
\text { Time: } 1205.8\end{array}$ & $\begin{array}{l}\text { Makespan:150. } \\
08 \\
\text { Time:2010 }\end{array}$ & $\begin{array}{l}\text { Makespan:193 } \\
.7 \\
\text { Time: } 2534\end{array}$ & $\begin{array}{l}\text { Makespan:240 } \\
.2 \\
\text { Time:3110.6 }\end{array}$ & $\begin{array}{l}\text { Makespan:274 } \\
\text { Time:3682 }\end{array}$ & $\begin{array}{l}\text { Makespan:401. } \\
98 \\
\text { Time:7821 }\end{array}$ & $\begin{array}{l}\text { Makespan:324 } \\
.9 \\
\text { Time:9320.2 }\end{array}$ \\
\hline $\begin{array}{l}\text { FAFS } \\
\text { A }\end{array}$ & $\begin{array}{l}\text { Makespan: } 118 \\
.8 \\
\text { Time: } 1200\end{array}$ & $\begin{array}{l}\text { Makespan:148. } \\
3 \\
\text { Time:2003 }\end{array}$ & $\begin{array}{l}\text { Makespan:190 } \\
\text { Time:2526 }\end{array}$ & $\begin{array}{l}\text { Makespan:237 } \\
.8 \\
\text { Time:3093 }\end{array}$ & $\begin{array}{l}\text { Makespan:270 } \\
.2 \\
\text { Time: } 3670\end{array}$ & $\begin{array}{l}\text { Makespan:400. } \\
6 \\
\text { Time:7806.5 }\end{array}$ & $\begin{array}{l}\text { Makespan:324 } \\
.1 \\
\text { Time:9312 }\end{array}$ \\
\hline
\end{tabular}




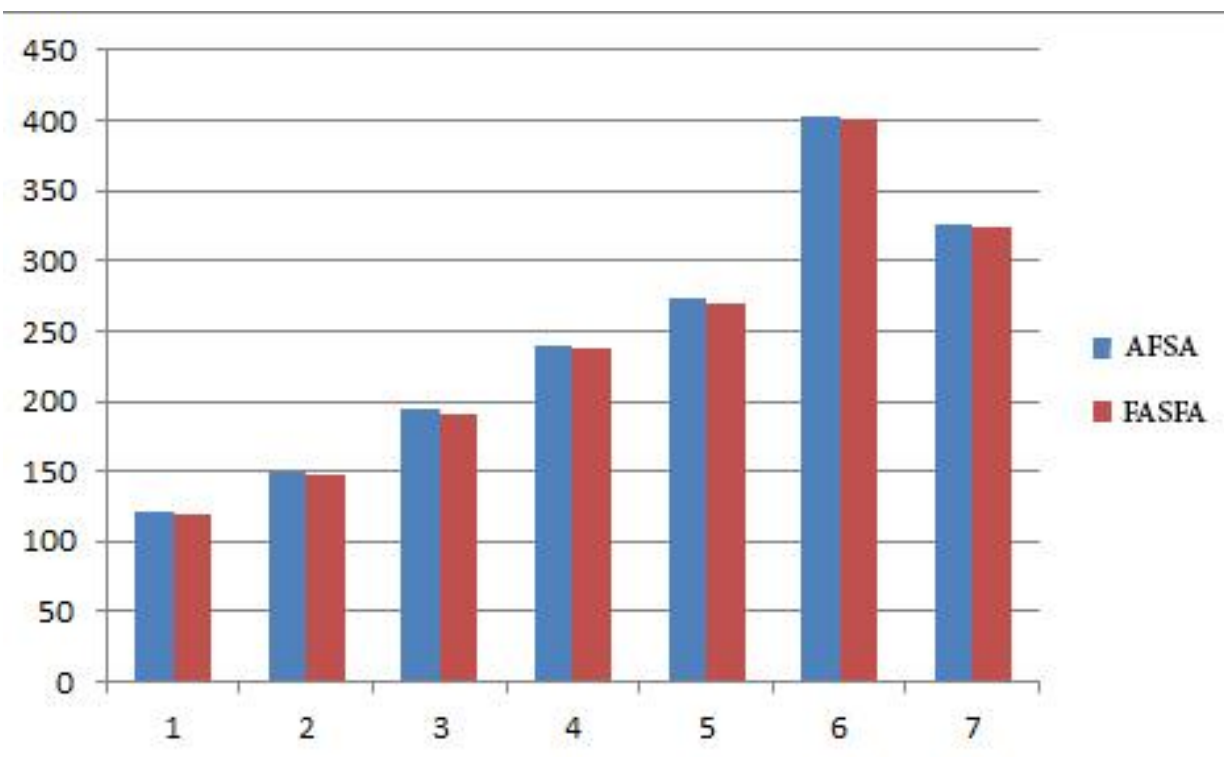

Figure 4 performance comparison between FASFA and AFSA

\section{REFERENCE}

[1] Arora, J., Introduction to Optimum Design, McGrawHill,1989

[2] Deb, K., Optimisation for Engineering Design, PrenticeHall, New Delhi.

[3] Glover, F. "Future Paths for Integer Programming and Links to Artificial Intelligence". Computers and Operations Research 13 (5): 533-549, 1986

[4] Farmer, J.D.; Packard, N.; Perelson, A., "The immune system, adaptation and machine learning". Physica D 22 (1-3): 187-204. 1986

[5] Goldberg, D. E., Genetic Algorithms in Search, Optimisation and Ma- chine Learning, Reading, Mass., Addison Wesley, 1989.

[6] Chu XiaoLi; Zhu Ying; Shi JunTao; Song JiQing, "Method of image segmentation based on Fuzzy CMeans Clustering Algorithm and Artificial Fish Swarm Algorithm," Intelligent Computing and Integrated Systems (ICISS), 2010 International Conference on, vol., no., pp.254,257, 22-24 Oct. 2010

[7] Tang Xueqin; DuanmuJingshun; Jin Liya; XuZongchang, "WNN optimization design based on Artificial FishSwarm Algorithm," Computer Science and Network Technology (ICCSNT), 2011 International Conference on , vol.4, no., pp.2747,2750, 24-26 Dec. 2011

[8] GaoZhong-hui; Ding Jian-yong; Liu Heng, "Hybrid algorithm based on artificial fish swarm algorithm and tabu search in distribution network reconfiguration," Computer Design and Applications (ICCDA), 2010 International Conference on , vol.5, no., pp.V5-415,V5-418, 25-27 June 2010

[9] Hai Ma; Yanjiang Wang, "An Artificial Fish Swarm Algorithm Based on Chaos Search," Natural Computation, 2009.ICNC '09. Fifth International Conference on, vol.4, no., pp.118,121, 14-16 Aug. 2009

[10]M A Awad, M Z Rashad, M AElsoud and M A Eldosuky. Article: Visualization of Job Scheduling in Grid
Computers. International Journal of Computer Applications 74(8):37-40, July 2013. Published by Foundation of Computer Science, New York, USA

[11] Jiang, M.Y., Yuan, D.F.: Artificial Fish Swarm Algorithm and Its Applications. Proc.Of International Conference on Sensing, Computing and Automation, Chongqing China, 2006, pp.1782-1787.

[12] Xiao, J.M., Zheng , X.M., Wang , X.H.: A Modified Artificial Fish-Swarm Algorithm. Proc. of IEEE the 6th World Congress on Intelligent Control and Automation, Dalian China, 2006, pp.3456-3460.

[13] Jiang, M.Y., Yuan, D.F.: Wavelet Threshold Optimization with Artificial Fish Swarm Algorithm. Proc. of IEEE International Conference on Neural Networks and Brain, Beijing China, 2005,pp.569 -572.

[14] Li, X.L.: A New Intelligent Optimization-Artificial Fish Swarm Algorithm. Doctor thesis, Zhejiang University of Zhejiang, China , 2003.

[15] MrSaeedFarzi "Efficient Job Scheduling in Grid Computing with Modified Artificial Fish Swarm Algorithm" International Journal of Computer Theory and Engineering, Vol. 1, No. 1, April 2009 1793-821X.

[16] Peter Mörters, Yuval Peres, Brownian Motion, Cambridge University Press, Mar 25, 2010.

[17] Andrej N. Borodin, PaavoSalminen, Handbook of Brownian Motion: Facts and Formulae, Springer, 2002.

[18] Brian H. Kaye,A Random Walk Through Fractal Dimensions, Second Edition, John Wiley \& Sons, Jul 11, 2008.

[19] Hans C. Foged by "Langevin equations For continuous time L'evy flights" 2008.

[20] Schoen, F., 'A wide class of test functions for global optimization', J. Global Optimization, 3, 133-137, 1993 
[21] Shang, Y. W., Qiu Y. H., 'A note on the extended rosenrbock function,' Evolutionary Computation, 14, 119-126., 2006

[22] Whitley, L. D., Mathias, K.E., Rana, S., \&Dzubera, J., Building better test functions, In Eshelman, L.J. (Ed), Proceeding of the Sixth International Conference on Genetic Algorithms, PP 239-246, Morgan Kaufmann, California, 1995.

[23] De Jong, K. A., An analysis of the behavior of a class of genetic adaptive systems, Doctoral dissertation, University of Michigan, Ann Arbor, University Microfilms No 76-9381, 1975.

[24] Rosenbrock, H.H., An Automatic method for finding the greatest or least value of a function, The Computer Journal, Vol. 3, No.3, PP 175-184, 1960.

[25] Dorigo, M,. Optimization, Learning and Natural Algorithms (Phd Thesis). Politecnico di Milano, Italie, 1992

[26] Karaboga, D., "An Idea Based On Honey Bee Swarm For Numerical Numerical Optimization". Technical ReportTR06 (Erciyes University, Engineering Faculty, Computer Engineering Department), 2005
[27] Kennedy, J.; Eberhart, R. "Particle Swarm Optimization". Proceedings of IEEE International Conference on Neural Networks. IV. pp. 1942-1948, 1995

[28] Gazi, K., and Passino, K. M., Stability analysis of social foraging swarms ,IEEE Trans. Sys. Man. Cyber. Part B Cybernetics, 34, 539-557, 2004.

[29] Glover, F. and M. Laguna. Tabu Search. Kluwer Academic Publishers, 1997.

[30] Hoos, H. H. and T. Stützle. Stochastic Local Search. Morgan Kaufmann, 2005

[31] M A Awad, M Z Rashad, M A Elsoud and M A Eldosuky. Article: FAFSA: Fast Artificial Fish Swarm Algorithm. International Journal of Information Science and Intelligent System, Vol. 2, No.1, 2013

[32]M A Awad, M Z Rashad, M AElsoud and M A Eldosuky. Article: Implementation of a New Visualization Framework of Job Scheduling in Grid Computers. International Journal of Computer Applications Volume 86 - No 5, January 2014. Published by Foundation of Computer Science, New York, USA 